\title{
PARAMETRIC WAVE-BREAKING ON STEEP REEFS
}

\author{
Shih-Feng Su${ }^{1}$, Alex Sheremet ${ }^{1}$ and Jane McKee Smith ${ }^{2}$
}

\begin{abstract}
A numerical model based on a nonlinear mild-slope equation, and modified to account for wave dissipation due to breaking is applied to investigate the transformation of the wave spectrum over a fringing reef. The three parameters $(\gamma, B, F)$ of the breaking model are calibrated for the best fit between the spectral shapes observed and modeled using an inverse modeling approach. The relationship between optimal values for $\gamma$ and $B$ derived from numerical simulations and other parameters characterizing wave and slope conditions (e.g., deep-water wave steepness, wave dispersivity, nonlinearity parameter) are investigated with the goal of formulating guidelines for the selection of adequate values. The results of this study disagree significantly with previously-proposed empirical relations between $\gamma$ and the deep-water wave steepness, but show good agreement with empirical relations relating $\gamma$ to other parameters. The breaking intensity parameter $B$ shows a largely linear dependency on the nonlinearity parameter.
\end{abstract}

Keywords: nonlinear waves; wave breaking; coral reef; wave modeling

\section{INTRODUCTION}

Islands surrounded by coral reefs are abundant in the tropical and subtropical regions. The form of fringing reefs provides natural protection landward from wave action. With increasing development of reef coasts, protecting coastal area from wave attack and providing habitat for marine ecology are a concern. Numerical modeling of wave transformation on reefs is essential for understanding reef processes such as the generation of infragravity waves, wave setup, and wave-induced reef-circulation. Modeling wave transformation on the reef should account for the rapid nonlinear wave shoaling and strong breaking-induced dissipation dominate the wave processes on the steep reef face.

Linear spectral models have been used with success on reefs to predict the dissipation of bulk (frequency integrated) wave energy dissipation due to wave breaking and bottom friction (Lowe et al., 2005; Massel and Gourlay, 2000). However, such models typically derive their success from finely tuned dissipation/growth parameterizations, and are unable to describe the change of spectral distribution of the energy flux, which is essentially a nonlinear process. Recent detailed field studies (Young, 1989; Hardy and Young, 1996; Samosorn and Woodroffe, 2008; Péquignet et al., 2009) have demonstrated the importance of nonlinear effects. Nonlinear spectral energy transfers from the spectral peak toward high frequencies is associated with the development of asymmetric and skewed wave profiles that drive sediments transport. Low-frequency infragravity (IG) waves with periods of the order of $1 \mathrm{~min}$, generated during shoaling (an essentially nonlinear process) can resonate slow waterlevel seiching of backreef lagoons (Péquignet et al., 2009), enhancing flooding during energetic wave events. On wide reef platforms, IG waves can entrain and transport sediments both across the reef flat and around the reef island (Samosorn and Woodroffe, 2008). These processes are beyond the capabilities of linear wave models, regardless of how accurate their wave-height predictions are.

The intense and localized wave breaking dominates dissipation processes on the reef face (Young, 1989; Gourlay, 1994). Parametric wave breaking models are commonly used to predict wave dissipation across the surf zone. Starting with the work of Thornton and Guza (1983), all available parameterizations depth-limited breaking wave height on beaches (Baldock et al., 1998; Kaihatu and Kirby, 1995; Janssen and Battjes, 2007, and others), are based on a number of standard parameters: $\gamma$ the ratio of breaking wave height to breaking depth, $B$ - the intensity of breaking. For nonlinear models that account for the evolution of the spectral shape, Kaihatu and Kirby (1995) introduced an additional parameter, $F$ - governing the frequency distribution of the dissipation rate. Empirical parameterizations developed over time for $\gamma$ are in general in good agreement on planar and barred beaches. The value of $\gamma$ is found to be dependent on the beach slope (Sallenger and Holman, 1985), offshore wave steepness (Battjes and Stive, 1985; Nairn, 1990) and non-dimensional depth kh (Ruessink et al., 2003). Most formulations were obtained from calibration with field observations and laboratory experiments for mild slopes (e.g., sandy bottom beaches).

For steep slopes with values of the order of $0.2-0.5$, characteristic of reefs, there is little available guidance for selecting optimal values for parameters $\gamma, B$, and $F$. In this paper, we use numerical

\footnotetext{
${ }^{1}$ Dept. of Civil and Coastal Engineering, Univ. of Florida, Gainesville, FL, 32611, USA

${ }^{2}$ Coastal \& Hydraulics Lab., US Army Engineer Research \& Development Center, Vicksburg, MS, 39180, USA
} 
simulation of wave spectral evolution over a fringing reef to estimate optimal values for $\gamma, B$, and $F$, and investigate their relation to the various wave conditions.

The numerical model implements the stochastic (phase-averaged) nonlinear (triad interaction) mild-slope equation developed by Agnon and Sheremet (1997), modified to incorporate the parametric wave breaking model proposed by Janssen and Battjes (2007). The observations used in this study are results of laboratory experiments conducted at University of Michigan at Ann Arbor (Demirbilek et al., 2007). The results of the simulations are compared with existing empirical parameterizations of wave breaking for application to reef profiles.

\section{MODEL FORMULATION}

\section{Nonlinear mild-slope equation model}

The nonlinear mild-slope equation was derived following the procedure outlined in Agnon and Sheremet (1997), modified to account for relatively large dissipation rates and steep slopes. Over a mild slope, the breaking process is spread over a relatively large area, and spectral dissipation rates are typically much smaller than the characteristic wave processes (e.g., Chen et al., 1997). In contrast, reefs are characterized by intense, localized breaking, with wave energy decaying typically by a factor of 5 within less than $100 \mathrm{~m}$ (Demirbilek et al., 2007). The model is a hyperbolic, frequency-domain, mild-slope equation model with triad wave-wave interactions. The wave transformation is modeled using the wave energy flux balance, which can be written as

$$
\begin{aligned}
& \frac{d \mathcal{F}_{j}}{d x}=-2 \kappa_{j} \mathcal{F}_{j} \\
& +8 \sum_{p, q>0}(W \delta \Re\{G\})_{j, p, q}\left(W_{j, p, q} \mathcal{F}_{p} \mathcal{F}_{q}+W_{j,-q, p} \mathcal{F}_{q} \mathcal{F}_{j}+W_{q,-p, j} \mathcal{F}_{p} \mathcal{F}_{j}\right) \\
& +16 \sum_{p, q>0}(W \delta \Re\{G\})_{j,-p, q}\left(W_{j,-p, q} \mathcal{F}_{p} \mathcal{F}_{q}+W_{p,-j, q} \mathcal{F}_{j} \mathcal{F}_{q}+W_{q, j, p} \mathcal{F}_{j} \mathcal{F}_{p}\right)
\end{aligned}
$$

where $x$ is the spatial coordinate, $\kappa_{j}$ is the dissipation rate of mode which takes into account energy losses due to wave breaking, $\delta$ is the Kronecker delta symbol, taking the value 1 or 0 according to $\omega_{j} \mp \omega_{p}-\omega_{q}$ being zero or nonzero. $\mathcal{F}_{j}$ is the averaged energy flux corresponding to the Fourier mode $j$ and defined as

$$
\mathcal{F}_{j}=\frac{1}{2}\left\langle\left|a_{j}\right|^{2} C_{j}\right\rangle,
$$

where $a_{j}$ is the complex amplitude, $C_{j}$ is the group velocity. The interaction coefficient $W_{j, \pm p, q}$ is a function of the frequencies and wave numbers of the interacting modes and given by

$$
W_{j, \pm p, q}=\frac{1}{8} \frac{\sqrt{g} \sigma_{j}}{\left(C_{j} C_{p} C_{q}\right)^{1 / 2} \sigma_{p} \sigma_{q}}\left[2 k_{ \pm p} k_{q}+\frac{\sigma_{ \pm p}}{\sigma_{j}} k_{q}^{2}+\frac{\sigma_{q}}{\sigma_{j}} k_{p}^{2}+\sigma_{p}^{2} \sigma_{q}^{2}-\sigma_{j}^{2} \sigma_{ \pm p} \sigma_{q}\right] \text { (3) }
$$

with $\sigma_{j}=\omega_{j} / \sqrt{g}, \omega_{j}$ is angular frequency, $\mathrm{g}$ is the gravitational acceleration and $k_{j}$ is the wave number. $\mathfrak{R}\{G\}$ is the real part of $G$, which is defined as

$$
G_{j, p, q}(x)=e^{-i \int_{-\infty}^{x} \Delta_{j, p, q} K d s} \int_{-\infty}^{x} e^{i \int_{-\infty}^{x} \Delta_{j, p, q} d s} d u
$$

where $\Delta K$ has an imaginary part representing dissipation

$$
\Delta_{j, \pm p, q} K=k_{j} \mp k_{ \pm p}-k_{q}-i\left(\kappa_{j}+\kappa_{p}+\kappa_{q}\right) .
$$

The numerical model requires knowledge of the power spectrum at offshore boundary for its integration. The measured time series at Sensor 2 (Figure 1) are used as the offshore boundary condition, which is analyzed using a standard fast Fourier transformation algorithm to obtain an initial wave spectrum. The initial wave spectrum is obtained by averaging over 68 sets of wave spectrum, 
each with 81 frequency modes and a frequency resolution $\Delta f=0.0049 \mathrm{~Hz}$ for a high frequency cutoff at $f=0.4 \mathrm{~Hz}$.

\section{Wave breaking model}

The evolution of wave energy across surf zone can be described by the one-dimensional wave energy flux balance equation

$$
\frac{d}{d x}\left(\frac{1}{2} \rho g \sum_{j=1} C_{j} \sum_{j=1}\left|a_{j}\right|^{2}\right)=-D,
$$

where $\rho$ is the water density and $D$ represents power dissipated per unit area. In application to random waves across the surf zone, Jassen and Battjes (2007) modified the wave breaking formulation of Baldock et al. (1998) to enhance the dissipation level on steep beaches, yielding

$$
D=\frac{B}{4 h} \rho g \bar{f} H_{r m s}^{3} Q_{b}
$$

in which $h$ is the water depth, $\bar{f}$ is the mean frequency, $H_{r m s}$ is the root-mean-square wave height, $B$ is a measure of intensity of breaking and $Q_{b}$ is the fraction of broken waves given by

$$
Q_{b}=\left[\left(R^{3}+\frac{3}{2} R\right) \exp \left(-R^{2}\right)+\frac{3}{4} \sqrt{\pi}(1-\operatorname{erf}(R))\right],
$$

where erf represents the error function and $R=\gamma h / H_{r m s}$ in which $\gamma$ is the ratio of wave height to water depth at initial breaking. Two free parameters $\gamma$ and $B$ indeed dominate wave breaking behavior. However, Mase and Kirby (1992), Kaihatu and Kirby (1995) and Chen et al. (1997) estimated a dissipation rate with a frequency-dependence in order to accommodate the empirical observations. The results were that energy is lost more strongly at higher frequencies. The wave breaking model can be modified to account for a frequency dependent mechanism, and the dissipation rate can be expressed as

$$
\kappa_{j}=\frac{B}{\sum_{j=1} C_{j}} \frac{\bar{f}}{h} H_{r m s} Q_{b}\left[F+(1-F) \frac{f_{j}^{2} \sum_{j=1}\left|a_{j}\right|^{2}}{\sum_{j=1} f_{j}^{2}\left|a_{j}\right|^{2}}\right],
$$

where $F$ is a weighting coefficient $(0 \leq F \leq 1)$. When $F=1$, the dissipation rate is independent on frequency.

\section{LABORATORY EXPERIMENTS}

Demirbilek et al. (2007) conducted laboratory experiments of shoaling and breaking of irregular waves over a fringing reef at the University of Michigan (UM). The laboratory study was conducted under the Surge and Wave Island Modeling Studies Program, to investigate the effect of wind on wave processes affecting the inundation of Pacific islands resulting from typhoons. A 1:64 scale profile representative of typical fringing reefs of the Pacific island of Guam was built in the wind-wave flume of $35 \mathrm{~m}$ length, $0.7 \mathrm{~m}$ width, and $1.6 \mathrm{~m}$ height. The corresponding prototype scale of the experimental setup is shown in Figure 1. The reef face consists of three sloping regions, with slopes of 1:5, 1:18.8, and 1:10.6, starting from offshore and ending at the reef edge. The reef top is wide and flat, meeting the beach slope at a slope of 1:12. In the present study, the model scale and Froude scaling are used to convert the measured data in the laboratory to equivalent prototype conditions for all calculations. The length scale factor is 64 and the velocity and time scale factors are 8, by Froude scaling. The incident waves were generated with a JONSWAP spectral shape with peak enhancement factor 3.3, offshore significant wave heights, $H_{0}$, ranging from 2.05 to $5.44 \mathrm{~m}$, and peak wave periods, $T_{\mathrm{p}}$, ranging from 8 to $20 \mathrm{sec}$. These wave tests represent extreme conditions associated with tropical cyclones. These test conditions were ran at four different still-water depths $(0 \mathrm{~m}, 1.02 \mathrm{~m}, 1.98 \mathrm{~m}$, and $3.2 \mathrm{~m}$ on the reef flat) to simulate conditions at different tide and surge levels. The still-water level used in this study is $3.2 \mathrm{~m}$, which is the highest water-level in the experiment. The incident wave conditions are listed in Table 1. Values of the corresponding deep-water wave steepness $S_{0}=H_{\mathrm{rms} 0} / L_{0}$ where $L_{0}=\mathrm{g} T_{\mathrm{p}}^{2} / 2 \pi$ and $H_{\mathrm{rms} 0}=1 / \sqrt{2} H_{0}$ are listed in the Table 1 . Breaker type is determined by the surf similarity number $\xi_{0}$ (Battjes, 1974), which is defined as: 


$$
\xi_{0}=\frac{\tan \beta}{\left(H_{0} / L_{0}\right)^{\frac{1}{2}}},
$$

where $\tan \beta$ is the slope of the bottom profile. Spilling breakers occur when $\xi_{0}<0.5$ and plunging breakers occur when $0.5<\xi_{0}<3.3$. All wave conditions considered in the present study are the plunging breaker type.

Nine capacitance-wire wave sensors were used to measure the water surface elevation (refer to Figure 1). Three sensors (Sensor 1-3) installed seaward of the reef face were arranged to allow separation of the incident and reflected wave trains. Three sensors (Sensor 4-6) were positioned over the reef slope. One sensor (Sensor 7) was installed at reef edge. The remaining two wave sensors (Sensor 8-9) were installed on the reef top. The sensors sampled at $20 \mathrm{~Hz}$ for $900 \mathrm{sec}$. The first 100 sec of data were neglected to allow waves to propagate through the gauge array. Spectra were estimated from zero-mean, 10\% cosine bell windowed wave records with band averaging. Resulting resolution bandwidth is $0.019 \mathrm{~Hz}$ and spectral estimates have a nominal 62 degrees of freedom.

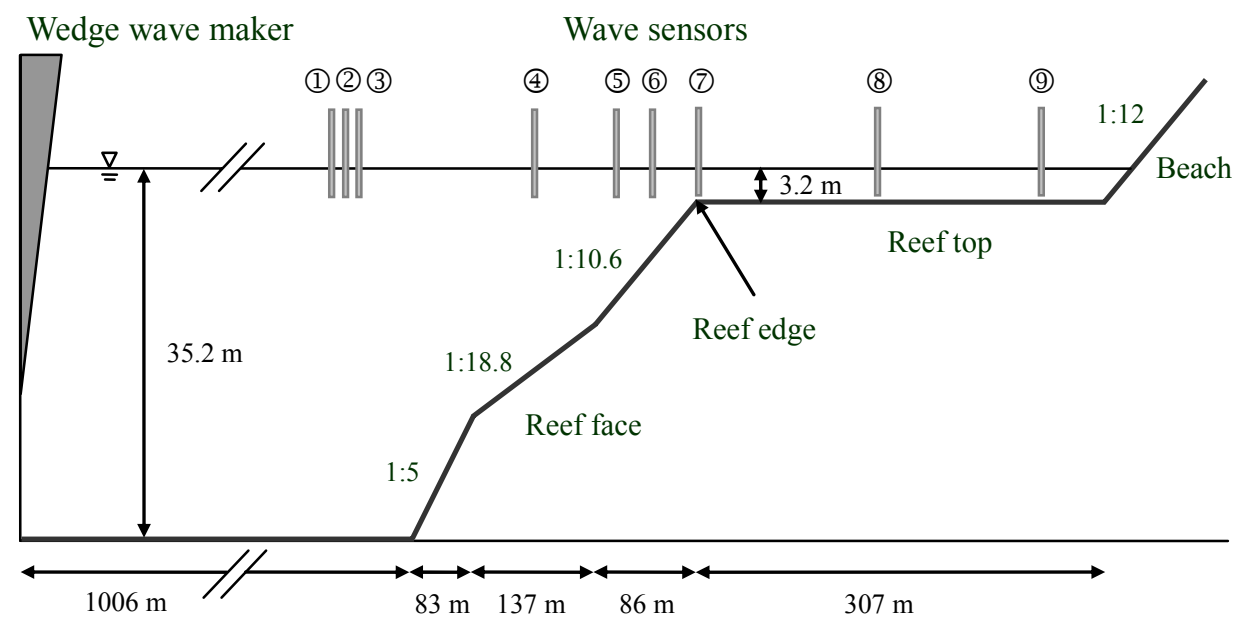

Figure 1. UM fringing reef experiment setup (Dimensions are in prototype scale).

\begin{tabular}{|c|c|c|c|c|}
\hline \multicolumn{5}{|c|}{ Table 1. Experimental wave conditions. } \\
\hline Case no. & $H_{0}(\mathrm{~m})$ & $T_{\mathrm{p}}(\mathrm{s})$ & $\mathrm{S}_{0}$ & $\xi_{0}$ \\
\hline 8_39_32 & 3.9 & 8 & 0.0276 & 0.478 \\
10_39_32 & 3.9 & 10 & 0.0177 & 0.596 \\
12_33_32 & 3.3 & 12 & 0.0106 & 0.770 \\
12_49_32 & 4.9 & 12 & 0.0156 & 0.636 \\
14_52_32 & 5.2 & 14 & 0.0120 & 0.723 \\
16_54_32 & 5.4 & 16 & 0.0099 & 0.797 \\
20_53_32 & 5.3 & 20 & 0.0057 & 1.054 \\
\hline
\end{tabular}

\section{CALIBRATION}

The effectiveness of the wave-breaking parameterization implemented in the model depends critically on the values of the three free parameters, $\gamma, B$ and $F$. Previous studies (e.g., Roelvink, 1993) based on linear models which require only two parameters $(\gamma$ and $B$ ) have typically assumed $B=1$ and found the optimal $\gamma$ through some calibration processes (typical value 0.7). For a nonlinear model, however, where the dissipation rate has a frequency distribution (Kaihatu and Kirby, 1995; Chen et al. 1997) and the value of $B=1$ might not represent accurately the physical processes. Different values for these two parameters may be appropriate also if the surf zone conditions or breaker types differ significantly from those for which they were originally proposed. In this study, the linear wave model (Eq. 1 excluding triad wave-wave interactions term) was used to calibrate parameters by minimizing the error between measured and modeled root-mean-square (rms) wave height, given as 


$$
e_{0}=\frac{1}{N} \sqrt{\sum_{n=1}^{N} \frac{\left[H_{r m s}^{\text {mea }}\left(x_{n}\right)-H_{r m s}^{\text {mod }}\left(x_{n}\right)\right]^{2}}{\left[H_{r m s}^{\text {mea }}\left(x_{n}\right)\right]^{2}}},
$$

where $N=4$ is the number of data points for tuning (Sensor 5,6,7 and 8), $H_{r m s}^{\text {mea }}$ and $H_{r m s}^{\bmod }$ are the measured and modeled rms wave height, respectively, and is defined as

$$
H_{r m s}^{\text {mea } / \mathrm{mod}}=\sqrt{8} \int_{f_{1}}^{f_{2}} S_{\text {mea } / \mathrm{mod}}(f) d f,
$$

where $S_{\text {mea }}$ and $S_{\text {mod }}$ represent the measured and modeled energy wave spectral densities, respectively. Linear estimates of bulk parameters such as significant wave heights typically ignore the energy in the IG band and use only frequencies $>0.02 \mathrm{~Hz}$ (swell and sea bands). However, the IG band plays an essential role in nonlinear shoaling and reach shoaling commonly involves energy transfers from the peak frequency to lower and higher frequencies. In the present study, low-frequency waves are assumed to be non-breaking. The numerical model excludes the dissipation in the infragravity waves $(f$ $<0.5 f_{\mathrm{p}}$ ). Therefore, the data are high-pass filtered with a low frequency cutoff $f_{1}=0.5 f_{\mathrm{p}}$ to exclude infragravity frequency, and low-pass filtered with a high frequency cutoff $f_{2}=0.4 \mathrm{~Hz}$.

A simple fit to a linear model to the observed decay of wave energy (or rms wave height) is not adequate, if wave-breaking dissipation has a strong frequency dependency, spectral transfers will affect the behavior of the system, e.g. by making more energy available to frequency bands where dissipation is stronger at higher frequencies. Therefore, we determined the optimal triplet $(\gamma, B, F)$ by fitting predicted spectra to the observed ones. Dissipation-rate weighting coefficient $F$ is calibrated with $(\gamma, B)$ pairs to minimize the error at high frequencies. The nonlinear wave model (including triad wave-wave interactions term) is used to calibrate by minimizing the rms error between the logarithms of measured and modeled spectral densities, given as

$$
e_{1}=\frac{1}{N}\left\{\sum_{n} \int_{f_{1}}^{f_{2}}\left[\log S_{\text {mea }}\left(x_{n}, f\right)-\log S_{\text {mod }}\left(x_{n}, f\right)\right]^{2} d f\right\}^{1 / 2} .
$$

\section{RESULTS AND DISCUSSION}

Figure 2 shows energy flux spectra $\left(H_{0}=3.9 \mathrm{~m}, T_{\mathrm{p}}=10 \mathrm{~s}\right)$ at the location o the sensors (see Figure 1). The numerical results use the parameterizations the optimal values of the wave-breaking parameters The evolution of the spectra shape is predicted well, including the transfer of energy from the peak to higher frequencies (e.g., Sensor 5 and Sensor 6), and also energy dissipation at reef flat (Sensor 7 and Sensor 8). The numerical results suggest that, to investigate the evolution of spectral energy on the reef, the spatial distribution of sensors in this experiment is not adequate, and that both the reef-face and the reef-top (e.g., inside the surf zone) should be more densely instrumented.

Figures 3 shows the cross-shore evolution of the normalized spectral flux $\left(H_{0}=3.9 \mathrm{~m}, T_{\mathrm{p}}=10 \mathrm{~s}\right)$ integrated over the entire frequency band, as well as for the sea, swell, and infragravity bands. The model accurately predicts evolution of energy flux in the swell frequency band. The observed energy flux in sea frequency band reaches its maximum at Sensor 6 and starts to decay. The model is able to capture the energy increase within the sea frequency band due to triad wave-wave interactions over reef face, and the decay induced by wave breaking onshore the reef edge. The energy flux in the infragravity band increases as waves propagate over the reef face as a result of nonlinear transfer of energy from the peak of the spectrum, and decay together with the sea and swell bands inside the surf zone near the reef edge. The model prediction of infragravity band spectral flux is less accurate due to limitations of model applicability related to very steep slopes, which are especially severe in the longwave range. Because the breaking dissipation parameterization is not applied to the infragravity band, its evolution is strictly driven by nonlinearities.

Existing empirical parameterizations of wave breaking are examined in order to study the adequateness for application to reef environments and extreme wave conditions. Parameterizations of $\gamma$ proposed by Battjes and Stive (1985)

$$
\gamma=0.5+0.4 \tanh \left(33 S_{0}\right),
$$

and Nairn (1990) 


$$
\gamma=0.39+0.56 \tanh \left(33 S_{0}\right)
$$

are as a function of offshore steepness. Figure 4 shows the variation of optimal values of $\gamma$ with offshore wave steepness for the data sets examined in this study. There is a clear discrepancy between optimal values of $\gamma$ and the existing parameterizations, especially for small offshore wave steepness, where optimal values of $\gamma$ are approximately twice the values suggested by previous parameterizations. The proposed empirical parameterizations decrease with decreasing offshore wave steepness, whereas the optimal values of $\gamma$ derived here using both linear model and the nonlinear models show an opposite trend.

Figure 5 shows the observed and calibrated values of $\gamma$ versus offshore wave steepness, compared with a monochromatic-wave formulation proposed by Singamsetti and Wind (1980):

$$
\gamma=\chi_{1}(\tan \beta)^{\chi_{2}}\left(\frac{H_{0}}{L_{0}}\right)^{\chi_{3}},
$$

where $\chi_{1}=0.937, \chi_{2}=0.155, \chi_{3}=-0.130$ are coefficients, calibrated for deepwater steepness $0.02<H_{0} / L_{0}<0.06$ and $1 / 40 \leq \tan \beta \leq 1 / 5$. This comparison also includes estimates of $\gamma$ derived directly from data, by assuming that the breaking point is at the reef edge (Sensor 7). The values of $\gamma$ estimated from data, derived using the numerical model, and based on equation (16) show the similar trend of increase with decreasing offshore steepness. This largely agrees with the results of Singamsetti and Wind (1980), for a tuned value of coefficient $\chi_{1}=0.62$ instead of the proposed $\chi_{1}=0.937$. Previous work also suggests that parameterizations based on offshore wave steepness (Battjes and Stive, 1985; Nairn, 1990) may have large errors, suggesting that in reef breaking wave conditions $\gamma$ is not uniquely determined by offshore wave steepness.

Figure 6 shows the optimal $\gamma$ values against the nondimensional water depth $k h$. The general trend of decreasing $\gamma$ with increasing $k h$ contradicts the parameterization of Ruessink et al. (2003), but it is in agreement with the relation proposed by Raubenheimer et al. (1996), who used a one-dimensional depth-averaged nonlinear water equation and field observations:

$$
\gamma=C_{0}+C_{1} \frac{\tan \beta}{k h},
$$

where $C_{0}$ and $C_{1}$ are adjustable coefficients. The coefficients have been fitted to optimal values of $\gamma$ by the nonlinear model, yielding the best fit values of $C_{0}=0.431, C_{1}=1.032$, with the correlation coefficient is 0.8524 . The reef-face slope is given with $\tan \beta=1 / 10.6$.

The dissipation rate trend should be examined with observations. Figure 7 shows observed dissipation rate versus frequency using the energy flux spectra $\left(H_{0}=4.9 \mathrm{~m}, T_{\mathrm{p}}=12 \mathrm{~s}\right)$ of consecutive wave sensors of $6,7,8,9$. Dissipation rates between Sensor 7 to 8 and Sensor 8 to 9 increase with increasing frequency (roughly as $f^{2}$ ). The inferred dissipation rates in the surf zone are shown to increase with increasing frequency, in qualitative agreement with Mase and Kirby (1992), Kaihatu and Kirby (1995) and Chen et al. (1997). The optimal values of $F$ are scattered over 0.5 to 0.65 . Kaihatu and Kirby (1995) predicted the spectral evolution on laboratory experiments data well using $F=0.5$.

Massel and Gourlay (2000) proposed that $B$ is closely related to the nonlinearity parameter $F_{\mathrm{c}}$ proposed by Nelson (1994)

$$
F_{c}=\frac{g^{1.25} H_{0}^{0.5} T^{2.5}}{h_{r}^{1.75}},
$$

where $g$ is the gravity acceleration, $T$ is the wave period and $h_{r}$ is the still water depth over the reef edge. $F_{\mathrm{c}}$ is proposed to be a suitable parameter in classification of wave transformation regimes over reefs. In particular, when $F_{\mathrm{c}}>150$, waves plunge on the reef edge and the amount of wave energy reaching the shore is small. When $F_{\mathrm{c}} \leq 100$, waves spill on the reef top, the greater part of energy is transmitted over the reef top. Figure 8 reveals the optimal $B$ values is dependent on $F_{\mathrm{c}}$. In the present study, $F_{\mathrm{c}}>150$ reveals waves start to break on the reef edge at which Sensor 7 is located. In other words, $B$ dominates breaking intensity from Sensor 7 to Sensor 8 . Values of $B$ were varied between 1.19 and 1.57 for the nonlinear model and between 1.28 and 2.2 for the linear model. Linear least squares fits through the values of $B$ is 


$$
B=b_{0}+b_{1} F_{c}
$$

where $b_{0}=1.245$ and $b_{1}=9.65 \times 10^{-5}$ for the linear model with the correlation coefficient 0.9281 and $b_{0}=1.169$ and $b_{1}=4.31 \times 10^{-5}$ for the nonlinear model with the correlation coefficient 0.8517 . It can be inferred that triad wave interaction dominates energy transfer, and affects the numerical values of $B$ (e.g., breaking intensity).
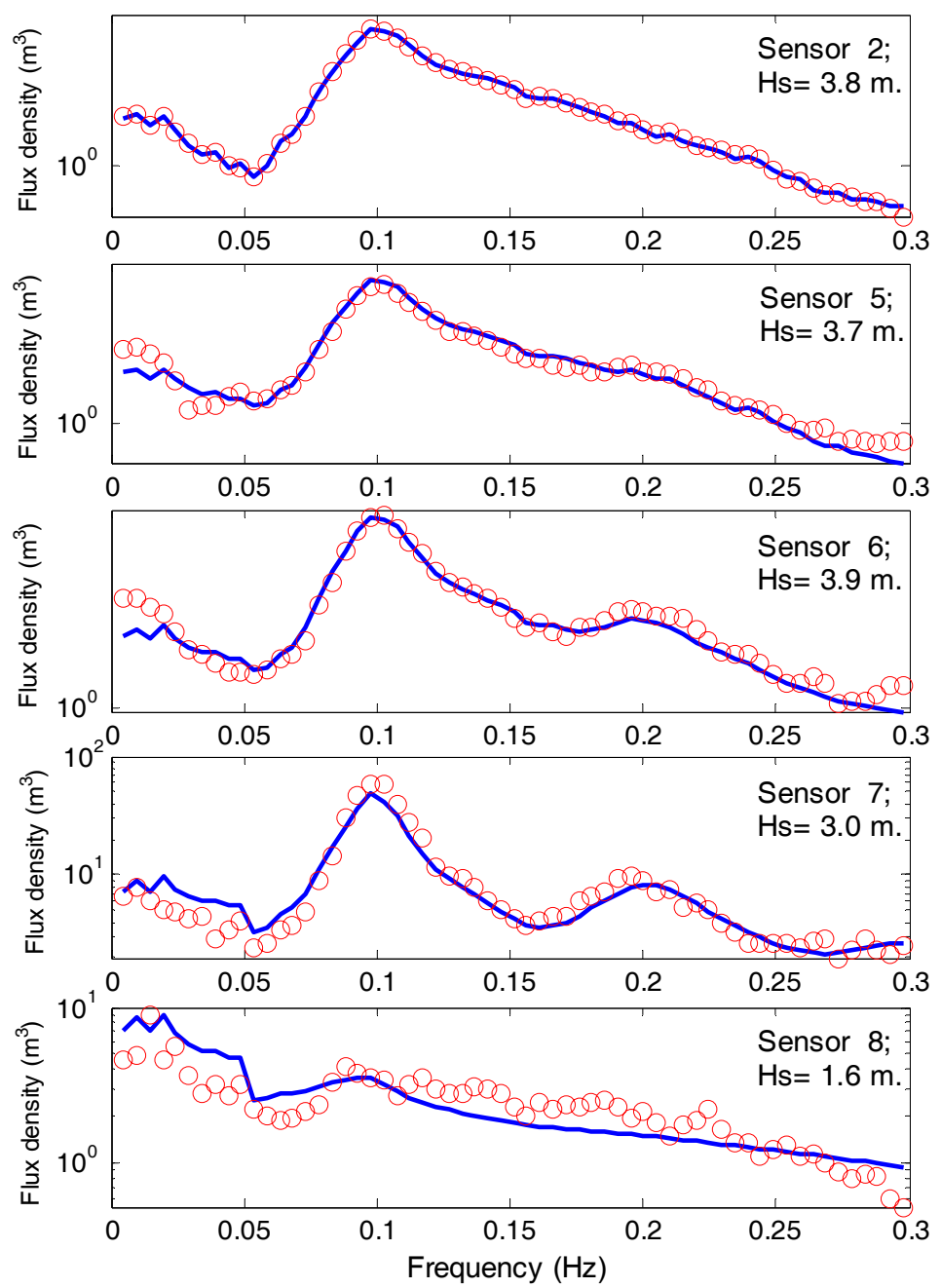

Figure 2. Energy flux spectral density at various locations of sensors. Measured spectra (circles), computed spectra (solid line). 


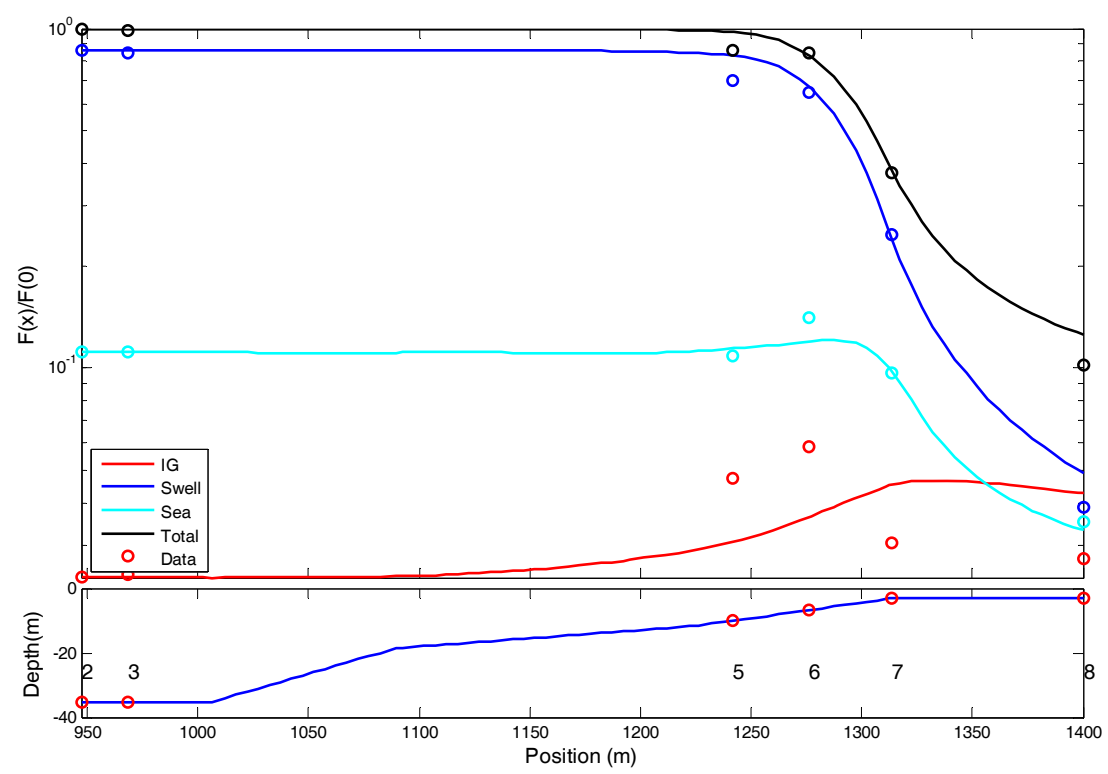

Figure 3. Cross-shore evolution of normalized spectral flux at upper panel. The curves are and circles represent model simulations on cross-shore and observations at sensor locations, respectively. The black denotes total spectral flux, the blue denotes swell-frequency band spectral flux, cyan denotes sea-frequency band spectral flux and red denotes infragravity-frequency band spectral flux. The reef profile with the location of sensors (red circles) is shown in the lower panel.

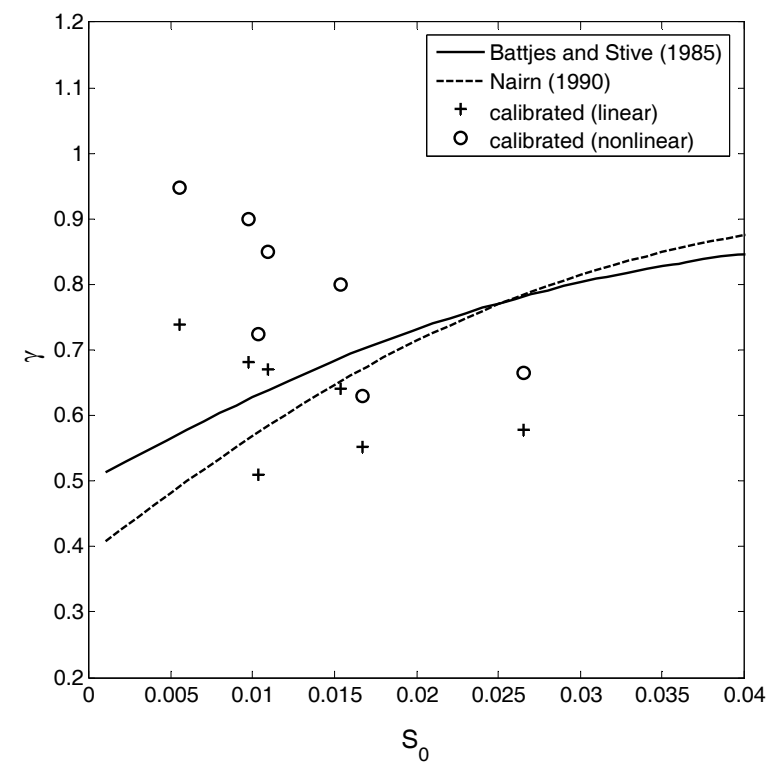

Figure 4. Calibrated $\gamma$ versus offshore wave steepness, $S_{0}$. Solid line denotes the parameterization of Battjes and Stive (1985); dashed line denotes the parameterization of Nairn (1990). Calibrations by the linear and nonlinear models are denoted by crosses and circles. 


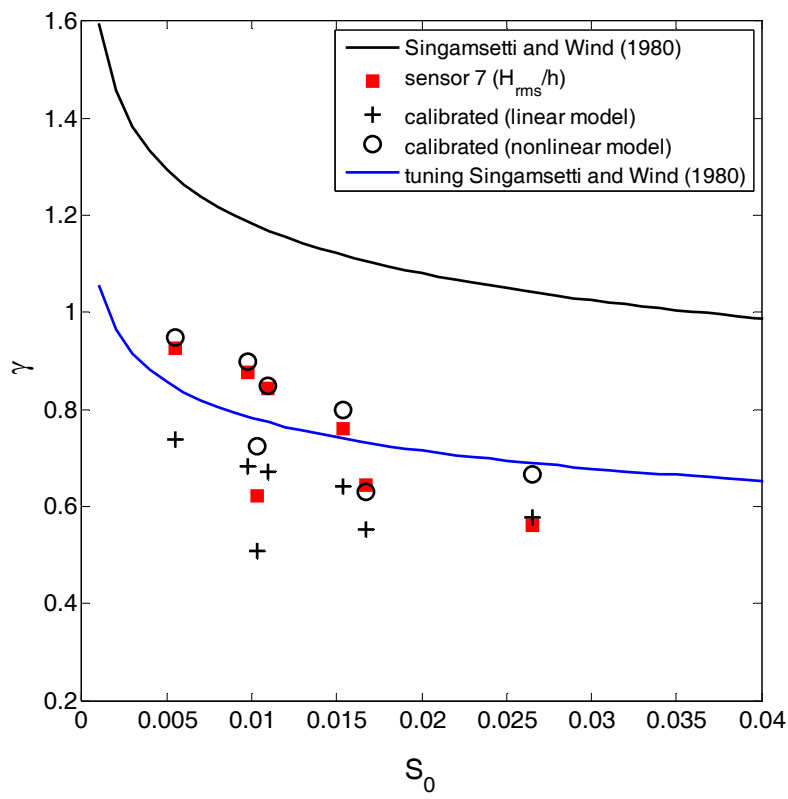

Figure 5. Observed and calibrated $\gamma$ versus offshore wave steepness, $\mathbf{S}_{0}$. Red squares denote measured values at the Sensor 7 (i.e., reef edge). Crosses and circles denote calibrated values based on the linear model and the nonlinear model, respectively. Black line is based on the parameterization of Singamsetti and Wind (1980), blue line is the tuned result of Singamsetti and Wind (1980).

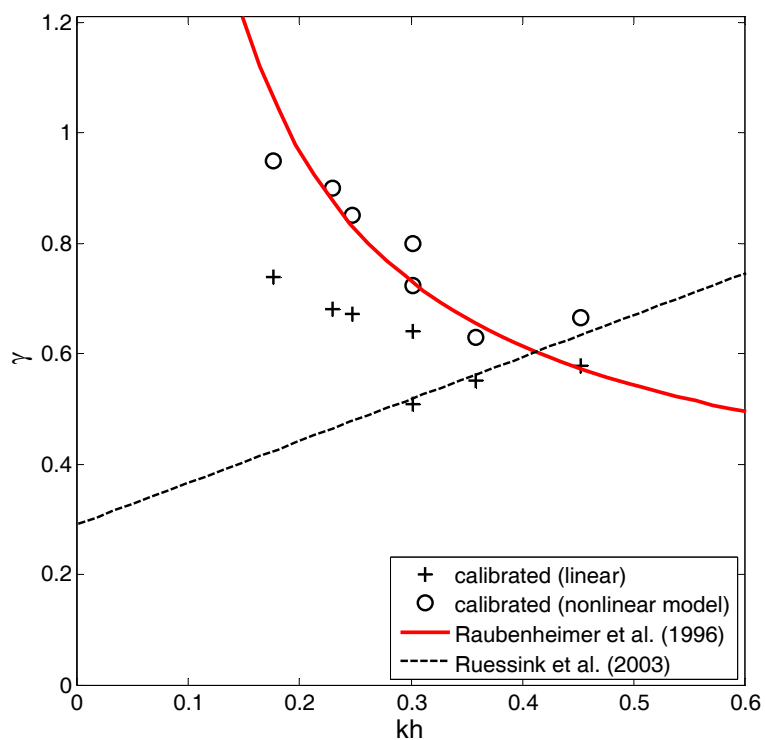

Figure 6. Calibrated $\gamma$ versus non-dimensional depth $\mathrm{kh}$. The dashed line denotes the parameterization by Ruessink et al. (2003). Solid red line denotes the relation proposed by Raubenheimer et al. (1996) with tuned coefficients. 


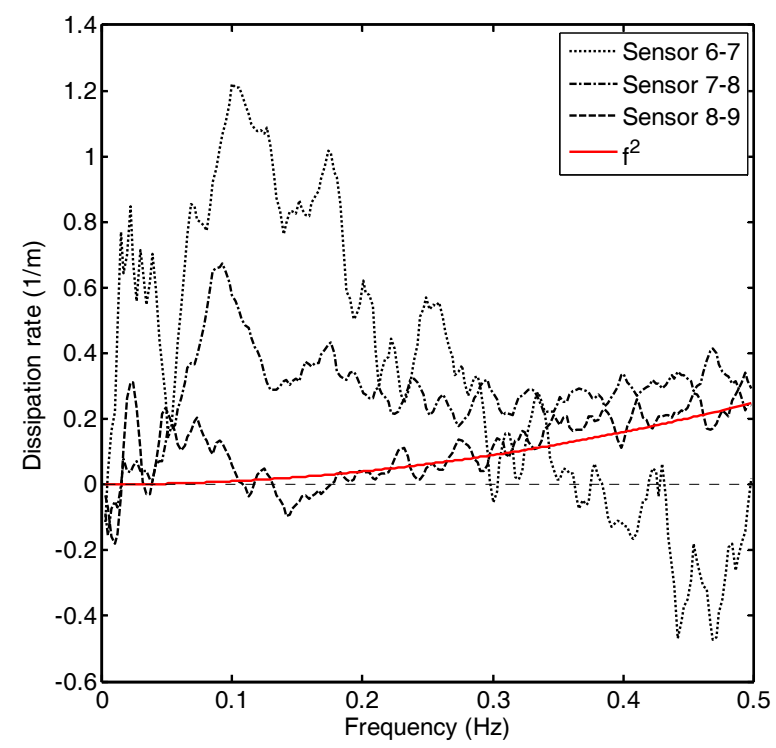

Figure 7. Energy dissipation rate versus frequency. The red line is proportional to $f^{2}$. The dissipation rate between two consecutive sensors is plotted with dotted (Sensor 6-7), dash-dotted (Sensor 7-8) and dashed (Sensor 7-8) line, respectively.

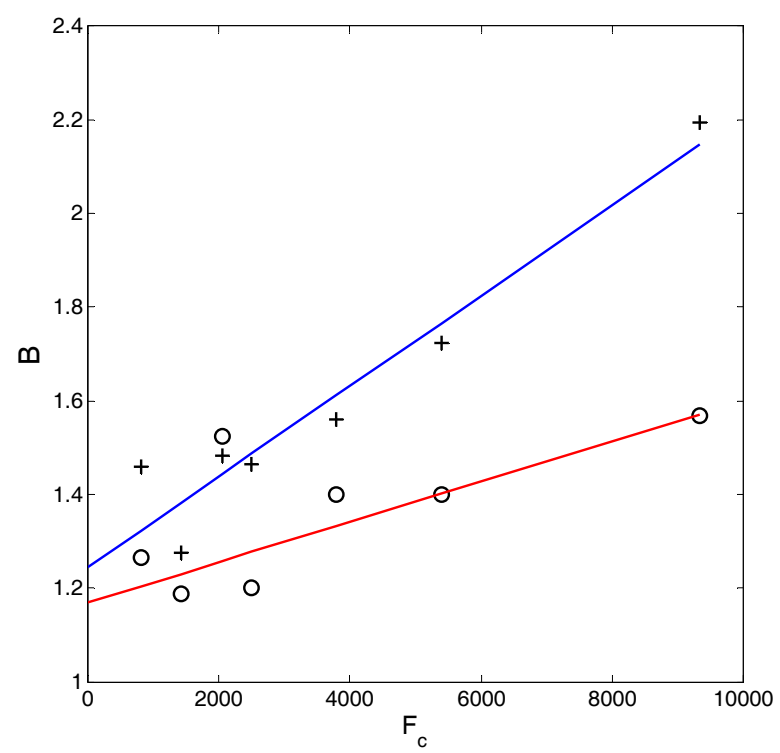

Figure 8. Optimal $B$ versus the nonlinearity parameter, $F_{c}$. Cross and circle points denote calibrated values by the linear wave model and the nonlinear wave model, respectively. The least squares linear fit of the calibrated values of $B$ using nonlinear model and linear model to $F_{c}$ are red line with the correlation and blue line, respectively.

\section{CONCLUSIONS}

In this study, a nonlinear wave model incorporating a parametric wave-breaking is applied to investigate the evolution of wave spectrum over a fringing reef. The set of free wave-breaking parameters $(\gamma, B, F)$ are obtained by minimizing the normalized root-mean-square error between measured and modeled total energy and spectral shapes. With the optimal values, good comparison of model predictions with observations in extreme wave conditions demonstrates the robustness of the 
model. The predicted evolution of the spectral shape agrees well with observations, including the development of the infragravity band and energy transfers to harmonics of the spectral peak.

The increase of infragravity wave energy flux during shoaling, an effect of nonlinear interaction well known on mild-slope beaches is also seen in laboratory reef experiments. Numerical simulations show that infragravity energy loss in the surf zone is related to the general decay of the sea-swell energy. This process might have several explanations, such as dissipation induced by surf turbulence, reverse shoaling (transfers of energy from IG to sea-swell) nonlinear effects, or even long-wave breaking. This effect is not fully understood, and deserves more research. The formulation of the model used here only provides insight into nonlinear effects, which do not appear to produce a strong enough reverse shoaling to explain it. In addition, the model has known limitations for severely steep slopes that affect the capabilities of the model in the IG frequency band.

Based on the results of the numerical modeling, the dependency of two wave-breaking parameters on various measures of nonlinearity and water depth are investigated. Numerically-derived optimal values of $\gamma$ disagree significantly with previously proposed empirical relations with offshore wave steepness, but showed good agreement with proposed relations with dispersion measures such as $k h$. The breaking-intensity parameter $B$ is approximately related to the nonlinearity parameter $F_{\mathrm{c}}$ which is related to the breaker type. The optimal values for the frequency distribution parameter $F$ are significantly different from the linear (default) value of 1 unity, suggesting that nonlinear wave evolution has significant effect on wave breaking. Our inverse modeling approach results in the relationship between optimal values of wave-breaking parameters and other parameters characterizing wave and slope conditions that provide formulating guidelines for selection of adequate values for predicting wave transformation on steep reefs.

\section{ACKNOWLEDGMENTS}

This study is supported by the US Army Corps of Engineers Research and Development Center, SWIMS Program, under Contract No. W912HZ-08-P-0346.

\section{REFERENCES}

Agnon, Y., and A. Sheremet. 1997. Stochastic nonlinear shoaling of directional spectra, J. Fluid Mech., 345, 79-99.

Baldock, T. E., P. Holmes, S. Bunker, and P. Van Weert. 1998. Cross-shore hydrodynamics within an unsaturated surf zone, Coast. Eng., 34, 173-196.

Battjes, J. A. 1974. Surf similarity, Proc. 14th Int. Conf. Coastal Engineering, ASCE, 466-480.

Battjes, J. A., and M. J. F. Stive. 1985. Calibration and verification of a dissipation model for random breaking waves, J. Geophys. Res., 90(C5), 9159-9167.

Chen, Y., R. T. Guza, and S. Elgar. 1997. Modeling spectra of breaking surface waves in shallow water, J. Geophys. Res., 102(C11), 25035-25046.

Demirbilek, Z., O. G. Nwogu, and D. L. Ward. 2007. Laboratory study of wind effect on runup over fringing reefs, Report 1: Data Report, ERDC/CHL-TR-07-4, Vickburg, MS: U.S. Army Engineer Research and Development Center.

Gourlay, M. R. 1994. Wave transformation on a coral reef, Coast. Eng., 23, 17-42.

Hardy, T. A., and I. R. Young. 1996. Field study of wave attenuation on an offshore coral reef, $J$. Geophys. Res., 101(C6), 14311-14326.

Janssen, T. T., and J. A. Battjes. 2007. A note on wave energy dissipation over steep beaches, Coast. Eng., 54(9), 711-716.

Kaihatu, J. M., and J. T. Kirby. 1995. Nonlinear transformation of waves in finite water depth, Phys. Fluids, 7, 1903-1914.

Lowe, R. J., J. L. Falter, M. D. Bandet, G. Pawlak, M. J. Atkinson, S. G. Monismith, and J. R. Koseff. 2005. Spectral wave dissipation over a barrier reef, J. Geophys. Res., 110, C04001, doi: 10.1029/2004JC002711.

Mase, H., and J. Kirby. 1992. Hybrid frequency-domain KdV equation for random wave transformation, Proc. 23rd Int. Conf. Coastal Engineering, ASCE, 474-482.

Massel, S. R., and M. R. Gourlay. 2000. On the modelling of wave breaking and set-up on coral reefs, Coast. Eng., 39, 1-27.

Nelson, R.C. 1994. Depth limited design wave heights in very flat regions, Coast. Eng. 23, 43-59.

Nairn, R. B. 1990. Prediction of cross-shore sediment transport and beach profile evolution, Ph.D. thesis, Imperial College, London, $391 \mathrm{pp}$. 
Péquignet, A. C. N., J. M. Becker, M. A. Merrifield, and J. Aucan. 2009. Forcing of resonant modes on a fringing reef during tropical storm Man-Yi, Geophys. Res. Lett., 36(L03607), doi: 10.1029/2008GL036259.

Raubenheimer, B., R. T. Guza, and S. Elgar. 1996. Wave transformation across the inner surf zone, $J$. Geophys. Res., 101, 25589-25597.

Roelvink, J. A. 1993. Dissipation in random wave groups incident on a beach, Coast. Eng., 19, $127-$ 150.

Ruessink, B. G., D. J. R. Walstra, and H. N. Southgate. 2003. Calibration and verification of a parametric wave model on barred beaches, Coast. Eng., 48, 139-149.

Sallenger, A. H., and R. A. Holman. 1985. Wave energy saturation on a natural beach of variable slope, J. Geophys. Res., 90, 11939-11944.

Samosorn, B., and C. D. Woodroffe. 2008. Nearshore wave environments around a sandy cay on a platform reef, Torres Strait, Australia, Cont. Shelf Res., 28, 2257-2274.

Singamsetti, S. R., and E. G. Wind. 1980. Breaking waves: characteristics of shoaling and breaking periodic waves normally incident to plane beaches of constant slope, 80 pp., Delft Hydraulic Laboratory.

Thornton, E. B., and R. T. Guza. 1983. Transformation of wave height distribution, J. Geophys. Res., 88(10), 5925-5938.

Young, I. R. 1989. Wave transformation over coral reefs, J. Geophys. Res., 94(C7), 9779-9789. 